\title{
Computation of fluxes of conservation laws
}

\author{
Alexei F. Cheviakov, \\ Department of Mathematics and Statistics, University of Saskatchewan, Saskatoon, S7N 5E6 Canada
}

\begin{abstract}
The direct method of construction of local conservation laws of partial differential equations (PDE) is a systematic method applicable to a wide class of PDE systems [Anco S. and Bluman G., Direct construction method for conservation laws of partial differential equations Part II: General treatment. Europ. J. Appl. Math. 13, 567-585 (2002)]. Within the direct method, one seeks multipliers, such that the linear combination of PDEs of a given system with these multipliers yields a divergence expression.

After local conservation law multipliers are found, one needs to reconstruct the fluxes of the conservation law. In this review paper, we discuss common methods of flux computation, compare them, and illustrate by examples. An implementation of these methods in symbolic software is also presented.
\end{abstract}

Keywords: Conservation laws; Direct construction method; Multipliers; Symbolic software.

\section{Introduction}

Local conservation laws of systems of partial differential equations (PDE), i.e., divergence expressions vanishing on solutions of a given PDE system, arise in a wide variety of applications and contexts. They often serve as mathematical expressions for fundamental physical principles, such as conservation of mass, momentum, charge, and energy. Conservation laws of time-dependent bounded systems describe conserved quantities. Conservation laws of ordinary differential equations (ODE) yield first integrals.

Conservation laws are widely used in PDE analysis, in particular, studies of existence, uniqueness and stability of solutions of nonlinear PDEs (e.g., [1-3]).

Another important area of application of conservation laws is the development and use of numerical methods. In particular, knowledge of conservation laws of a PDE system of interest enables one to draw from a wide variety of robust and efficient numerical schemes which have been derived in the last decades, including finite volume, finite element, and discontinuous Galerkin methods (see, e.g., [4, Chapter II; 5, Chapter IV]).

In addition, local conservation laws of a given PDE system are used to introduce nonlocal (potential) variables and thus construct PDE systems nonlocally related to a given one, with the same solution set. The related framework of nonlocally related systems (e.g., [6-11]) has yielded many new results over the recent years.

When a PDE system has an infinite set of local conservation laws involving arbitrary functions, it can be sometimes mapped into a linear PDE system by an invertible transformation, which can

\footnotetext{
a) Electronic mail: cheviakov@math.usask.ca
} 
be derived explicitly when it exists [12]. An infinite countable set of local conservation laws is often associated with integrability.

In the current paper, we consider local divergence-type conservation laws. Other types of conservation laws (such as lower-degree, e.g., curl-type, and nonlocal conservation laws) also arise in applications.

We now turn to the question of derivation of conservation laws. Many classical conservation laws have been found by ad hoc methods. One of the most well-known systematic methods is due to Emmy Noether, who demonstrated that for self-adjoint (variational) PDE systems, conservation laws arise from variational symmetries, i.e., symmetries that preserve the action integral [13]. However, the applicability of Noether theorem is severely limited, since the majority of PDE systems arising in applications are not self-adjoint [14].

An interesting review devoted to comparison of several methods of computation of conservation laws is found in [15].

A more general systematic method of constructing local conservation laws called the direct method was suggested in [16,17] (see also [18]). We review this method and the underlying theory in Section 3 of the current paper. Within this method, one seeks a set of local multipliers (also called factors or characteristics) depending on independent and dependent variables of a given PDE system and derivatives of dependent variables up to some fixed order, such that a linear combination of the PDEs of the system system taken with these multipliers yields a divergence expression. Families of multipliers that yield conservation laws are found from determining equations that follow from Euler differential operators. After finding sets of local conservation law multipliers, one needs to derive expressions for the corresponding conservation law fluxes.

The goal of this paper is to review and illustrate available methods of flux construction (Section 4). We start from a direct ("brute force") method (Section 4.1) that converts a conservation law directly into the set of determining equations (linear PDEs) for the unknown fluxes. This method is easy to apply in the case of simple conservation laws, including those involving arbitrary functions.

The second and the third methods (Sections 4.2 and 4.3) employ integral formulas, related to homotopy operators, to compute the fluxes. These formulas can be used to produce closed-form flux expressions for rather complicated forms of conservation laws, except for the cases when the conservation law involves arbitrary functions. The first integral formula is presented following [19]. The second integral formula initially appeared in [17], but is re-derived in the present paper in a different form, which is symmetric with respect to independent variables, and is simpler for practical computations and software implementation.

The fourth method [20] (Section 4.4) applies to scaling-homogeneous conservation laws of scalinginvariant PDE systems, which often occur in applications. Within this method, one finds fluxes of conservation laws through a rather simple formula involving no integration.

Another appropriate reference for the above four methods is [21, Section 1.3].

In Section 5, we briefly compare and discuss the four flux computation methods presented in the current paper.

Finally, in Section [6 we present a symbolic software implementation of the direct method of conservation law construction and the four flux computation methods in the symbolic package GeM for Maple [22], and illustrate these methods with examples.

There also exist other symbolic software packages implementing the direct method, as well as 
other methods for computation of conservation laws; see [19,26-29] and references therein.

\section{Notation and definitions}

\section{General definitions}

Consider a general system of $N$ partial differential equations of order $k$ with $n$ independent variables $x=\left(x^{1}, \ldots, x^{n}\right)$ and $m$ dependent variables $u(x)=\left(u^{1}(x), \ldots, u^{m}(x)\right)$, denoted by

$$
R^{\sigma}[u]=R^{\sigma}\left(x, u, \partial u, \ldots, \partial^{k} u\right)=0, \quad \sigma=1, \ldots, N .
$$

Here partial derivatives are denoted by $u_{i}^{\mu}=\frac{\partial u^{\mu}(x)}{\partial x^{i}}$;

$$
\partial u \equiv \partial^{1} u=\left(u_{1}^{1}(x), \ldots, u_{n}^{1}(x), \ldots, u_{1}^{m}(x), \ldots, u_{n}^{m}(x)\right)
$$

denotes the set of all first-order partial derivatives;

$$
\begin{aligned}
\partial^{p} u & =\left\{u_{i_{1} \ldots i_{p}}^{\mu} \mid \mu=1, \ldots, m ; i_{1}, \ldots, i_{p}=1, \ldots, n\right\} \\
& =\left\{\frac{\partial^{p} u^{\mu}(x)}{\partial x^{i_{1}} \ldots \partial x^{i_{p}}} \mid \mu=1, \ldots, m ; i_{1}, \ldots, i_{p}=1, \ldots, n\right\}
\end{aligned}
$$

denotes the set of all partial derivatives of order $p$.

Within the current paper, the notation " $f[u]$ " means that $f$ is a function of one or more independent variables $x$, dependent variables $u$, and possibly derivatives of dependent variables, up to some fixed order, i.e.,

$$
f[u] \equiv f\left(x, u, \partial u, \ldots, \partial^{l} u\right), \quad l \geq 0 .
$$

Definition 1. The given PDE system (2.1) is written in a solved form with respect to some leading derivatives, if

$$
R^{\sigma}[u]=u_{i_{\sigma, 1} \ldots i_{\sigma, s}}^{j_{\sigma}}-G^{\sigma}\left(x, u, \partial u, \ldots, \partial^{k} u\right)=0, \quad \sigma=1, \ldots, N
$$

where $s \leq k, 1 \leq j_{\sigma} \leq m, 1 \leq i_{\sigma, 1}, \ldots, i_{\sigma, s} \leq n$ for all $\sigma=1, \ldots, N$. In (2.2),$\left\{u_{i_{\sigma, 1} \ldots i_{\sigma, s}}^{j_{\sigma}}\right\}$ is a set of $N$ linearly independent $s$ th order leading partial derivatives, with the property that none of them or their differential consequences appears in $\left\{G^{\sigma}[u]\right\}_{\sigma=1}^{N}$. (For example, for dynamical PDE systems, time derivatives yield a natural set of leading derivatives.)

Definition 2. A PDE system (2.1) is in Cauchy-Kovalevskaya form with respect to an independent variable $x^{j}$, if the system is in the solved form for the highest derivative of each dependent variable with respect to $x^{j}$, i.e.,

$$
\frac{\partial^{s}(\sigma)}{\partial\left(x^{j}\right)^{s}(\sigma)} u^{\sigma}=G^{\sigma}\left(x, u, \partial u, \ldots, \partial^{k} u\right), \quad 1 \leq s_{(\sigma)} \leq k, \quad \sigma=1, \ldots, m,
$$

where all derivatives with respect to $x^{j}$ appearing in the right-hand side of each PDE of (2.3) are of lower order than those appearing on the left-hand side. 
Definition 3. A PDE system (2.1) admits a Cauchy-Kovalevskaya form if it can be written in Cauchy-Kovalevskaya form (2.3) with respect to some independent variable (after a point (contact) transformation if necessary).

A PDE system can admit a Cauchy-Kovalevskaya form only if its number of dependent variables equals the number of PDEs in the system, i.e., $N=m$. Many PDE systems arising in applications admit a Cauchy-Kovalevskaya form with respect to one or more of its independent variables. PDE systems that do not admit a Cauchy-Kovalevskaya form are typically PDE systems with differential constraints, such as Maxwell's equations.

Definition 4. The total derivative operators with respect to independent variables are given by

$$
\mathrm{D}_{i}=\frac{\partial}{\partial x^{i}}+u_{i}^{\mu} \frac{\partial}{\partial u^{\mu}}+u_{i i_{1}}^{\mu} \frac{\partial}{\partial u_{i_{1}}^{\mu}}+u_{i i_{1} i_{2}}^{\mu} \frac{\partial}{\partial u_{i_{1} i_{2}}^{\mu}}+\ldots, \quad i=1, \ldots, n .
$$

In (2.4) and below, summation over repeated indices is assumed.

Definition 5. The differential Euler operator with respect to a function $U(x)$ is defined by

$$
\mathrm{E}_{U}=\frac{\partial}{\partial U}-\mathrm{D}_{i} \frac{\partial}{\partial U_{i}}+\ldots+(-1)^{s} \mathrm{D}_{i_{1}} \ldots \mathrm{D}_{i_{s}} \frac{\partial}{\partial U_{i_{1} \ldots i_{s}}}+\ldots
$$

\section{Local conservation laws}

Definition 6. A local conservation law of PDE system (2.1) is a divergence expression

$$
\operatorname{div} \boldsymbol{\Phi}[u] \equiv \mathrm{D}_{i} \Phi^{i}[u] \equiv \mathrm{D}_{1} \Phi^{1}[u]+\ldots+\mathrm{D}_{n} \Phi^{n}[u]=0
$$

holding for all solutions of PDE system (2.1). In (2.6) $, \Phi^{i}[u]=\Phi^{i}\left(x, u, \partial u, \ldots, \partial^{r} u\right), i=1, \ldots, n$ are called the fluxes of the conservation law, and the highest order derivative $(r)$ present in the fluxes $\Phi^{i}[u]$ is called the (differential) order of a conservation law.

If one of the independent variables of the PDE system (2.6) is time $t$, the conservation law (2.6) takes the form

$$
\mathrm{D}_{t} \Psi[u]+\operatorname{div} \boldsymbol{\Phi}[u]=0
$$

where $\operatorname{div} \boldsymbol{\Phi}[u]=\mathrm{D}_{i} \Phi^{i}[u]=\mathrm{D}_{1} \Phi^{1}[u]+\ldots+\mathrm{D}_{n-1} \Phi^{n-1}[u]$ is a spatial divergence, and $x=$ $\left(x^{1}, \ldots, x^{n-1}\right)$ are $n-1$ spatial variables. Here $\Psi[u]$ is referred to as a density, and $\Phi^{i}[u]$ as spatial fluxes of the conservation law (2.7).

Definition 7. A local conservation law (2.6) of the PDE system (2.1) is trivial if its fluxes are of the form $\Phi^{i}[u]=M^{i}[u]+H^{i}[u]$, where $M^{i}[u]$ vanishes on the solutions of the system (2.1), and $\mathrm{D}_{i} H^{i}[u] \equiv 0$ is a trivial divergence.

Definition 8. Two local conservation laws (2.1) are equivalent if they differ by a trivial conservation law. Similarly, a set of local conservation laws of the form (2.1) is linearly dependent if its nontrivial linear combination (i.e., a linear combination with coefficients which are not all equal to zero) yields a trivial conservation law. 


\section{The direct method of construction of conservation laws}

For a given PDE system, one is interested in finding its nontrivial linearly independent local conservation laws.

We now outline a systematic and generally applicable method of construction of local conservation laws of PDE systems, called the direct method [16,17].

\subsection{Multipliers. The sequence of the direct method}

Consider a set of multipliers $\left\{\Lambda_{\sigma}[U]\right\}_{\sigma=1}^{N}=\left\{\Lambda_{\sigma}\left(x, U, \partial U, \ldots, \partial^{l} U\right)\right\}_{\sigma=1}^{N}$ which, taken as factors in the linear combination of equations of the PDE system (2.1), yield a divergence expression

$$
\Lambda_{\sigma}[U] R^{\sigma}[U] \equiv \mathrm{D}_{i} \Phi^{i}[U]
$$

which holds for arbitrary functions $U(x)$. Then on the solutions $U(x)=u(x)$ of the PDE system (2.1), one has a local conservation law

$$
\Lambda_{\sigma}[u] R^{\sigma}[u]=\mathrm{D}_{i} \Phi^{i}[u]=0
$$

provided that each multiplier is non-singular. [A multiplier $\Lambda_{\sigma}[U]$ is called singular if it is a singular function when computed on solutions $U(x)=u(x)$ of a given PDE system (2.1) (e.g., $\left.\Lambda_{\sigma}[U]=F[U] / R^{\sigma}[U]\right)$. In practice, one is interested only in non-singular sets of multipliers, since singular multipliers can yield arbitrary divergence expressions which are not conservation laws of the given PDE system. For example, taking $\Lambda_{\sigma_{1}}[U]=\operatorname{div} \boldsymbol{\Phi}[U] / R^{\sigma_{1}}[U]$ and $\Lambda_{\sigma \neq \sigma_{1}}[U]=0$, one obtains $\Lambda_{\sigma}[U] R^{\sigma}[U]=\operatorname{div} \boldsymbol{\Phi}[U]$, which does not yield a conservation law of the PDE system (2.1).]

To seek sets of multipliers $\left\{\Lambda_{\sigma}[U]\right\}_{\sigma=1}^{N}$ that yield conservation laws (3.2) of a given PDE system (2.1), one uses the following fundamental property of Euler operators (2.5).

Theorem 1. Let $x=\left(x^{1}, \ldots, x^{n}\right)$ and $U(x)=\left(U^{1}(x), \ldots, U^{m}(x)\right)$. An expression $f[U]=$ $f\left(x, U, \partial U, \ldots, \partial^{l} U\right), l \geq 0$, is annihilated by all Euler operators $\mathrm{E}_{U^{j}}$, i.e.,

$$
\mathrm{E}_{U^{j}} f[U] \equiv 0, \quad j=1, \ldots, m,
$$

if and only if $f[U]$ is a divergence expression

$$
f[U]=\mathrm{D}_{i} \Phi^{i}[U]
$$

for some set of fluxes $\left\{\Phi^{i}[U]\right\}_{i=1}^{n}=\left\{\Phi^{i}\left(x, U, \partial U, \ldots, \partial^{r} U\right)\right\}_{i=1}^{n}$.

Corollary 1. A set of non-singular multipliers $\left\{\Lambda_{\sigma}\left(x, U, \partial U, \ldots, \partial \partial^{l} U\right)\right\}_{\sigma=1}^{N}$ yields a local conservation law for the PDE system (2.1) if and only if

$$
\mathrm{E}_{U^{j}}\left(\Lambda_{\sigma}\left(x, U, \partial U, \ldots, \partial^{l} U\right) R^{\sigma}\left(x, U, \partial U, \ldots, \partial^{k} U\right)\right)=0, \quad j=1, \ldots, m,
$$

hold for arbitrary functions $U(x)$. 


\section{The sequence of the direct method}

1. For a given PDE system (2.1), seek multipliers of the form $\left\{\Lambda_{\sigma}\left(x, U, \partial U, \ldots, \partial^{l} U\right)\right\}_{\sigma=1}^{N}$, for some specified order $l$. Choose the dependence of multipliers on their arguments so that singular multipliers do not arise.

2. Solve the set of determining equations (3.4) for arbitrary $U(x)$ to find all such sets of multipliers.

3. Find the corresponding fluxes $\Phi^{i}[U]=\Phi^{i}\left(x, U, \partial U, \ldots, \partial^{r} U\right)$ satisfying the identity (3.1).

4. Each set of fluxes and multipliers yields a local conservation law

$$
\mathrm{D}_{i} \Phi^{i}\left(x, u, \partial u, \ldots, \partial^{r} u\right)=0
$$

holding for all solutions $u(x)$ of the given PDE system (2.1).

In practical computations, when it is possible, it is convenient to write the given PDE system (2.1) in a solved form (2.2) with respect to some leading derivatives. Then in Step 1 of the direct method, one can easily avoid singular conservation law multipliers, by excluding such leading derivatives and their differential consequences from the multiplier dependence.

\subsection{Completeness of the direct method}

The direct method outlined above can be applied to a wide class of PDE systems. Indeed, the only requirement is the existence of all derivatives that arise in the determining equations (3.4).

However, for a given PDE system, it is often important to know whether or not the direct method can yield all its nontrivial local conservation laws (at least up to some fixed order). Conversely, under what conditions does a set of local multipliers yield only nontrivial local conservation laws? We now outline the results related to these questions.

When a given PDE system is written in a solved form, one can show that the application of the direct method with multipliers of sufficiently general dependence yields all local conservation laws of the given PDE system of any fixed order order $l$. In particular, the following theorem holds [21].

Theorem 2. For each local conservation law $\mathrm{D}_{i} \Phi^{i}[u]=0$ of a PDE system (2.1) written in a solved form (2.2), there exists an equivalent local conservation law $\mathrm{D}_{i} \widetilde{\Phi}^{i}[u]=0$ which can be expressed in $a$ characteristic form

$$
\mathrm{D}_{i} \widetilde{\Phi}^{i}[U]=\widetilde{\Lambda}_{\sigma}[U]\left(U_{i_{\sigma, 1 \ldots i} i_{\sigma, s}}^{j_{\sigma}}-G^{\sigma}[U]\right)
$$

in terms of a set of non-singular local multipliers $\left\{\widetilde{\Lambda}_{\sigma}[U]\right\}_{\sigma=1}^{N}$, with fluxes that contain no leading derivatives $U_{i_{\sigma, 1 \ldots} \ldots i_{\sigma, s}}^{j_{\sigma}}$ nor their differential consequences.

However, even when a PDE system is written in a solved form (2.2), the correspondence between equivalence classes of conservation laws and sets of conservation law multipliers might not be oneto-one. The following theorem holds [17]. 
Theorem 3. Suppose a PDE system (2.1) admits a Cauchy-Kovalevskaya form (2.3). Then all of its non-trivial (up to equivalence) local conservation laws arise from multipliers. Moreover, there is a one-to-one correspondence between equivalence classes of conservation laws and sets of conservation law multipliers $\left\{\Lambda_{\sigma}[U]\right\}_{\sigma=1}^{N}$, if the multipliers do not involve derivatives with respect to $x^{j}$.

\section{Computation of fluxes of conservation laws}

Consider a set of non-singular multipliers $\left\{\Lambda_{\sigma}[U]=\Lambda_{\sigma}\left(x, U, \partial U, \ldots, \partial^{l} U\right)\right\}_{\sigma=1}^{N}$ that yields a divergence expression (3.1) for a PDE system (2.1) for some set of fluxes $\left\{\Phi^{i}[U]=\Phi^{i}\left(x, U, \partial U, \ldots, \partial^{r} U\right)\right\}_{i=1}^{n}$. Hence one has a local conservation law (3.2) holding on the solutions $U(x)=u(x)$ of the PDE system (2.1).

Given a set of conservation law multipliers, the problem of finding the fluxes $\left\{\Phi^{i}[U]\right\}_{i=1}^{n}$ is formally a problem of inversion of the divergence differential operator. Even modulo trivial conservation laws of the first type (Definition [7, $H^{i}[u] \equiv 0$ ), a set of multipliers defines an equivalence class of conservation laws, up to free constants and arbitrary functions. For example, suppose in a three-dimensional space $(x, y, z)(n=3)$, the fluxes of a conservation law

$$
\sum_{\sigma=1}^{N} \Lambda_{\sigma}[U] R^{\sigma}[U] \equiv \sum_{i=1}^{3} \mathrm{D}_{i} \Phi^{i}[U]
$$

are given by

$$
\mathbf{\Phi}[U]=\left(\Phi^{1}[U], \Phi^{2}[U], \Phi^{3}[U]\right)=\operatorname{div}^{-1}\left(\sum_{\sigma=1}^{3} \Lambda_{\sigma}[U] R^{\sigma}[U]\right) .
$$

Then the expression

$$
\widehat{\mathbf{\Phi}}[U]=\mathbf{\Phi}[U]+\operatorname{curl} \mathbf{K},
$$

for an arbitrary smooth vector field $\mathbf{K}$, also yields fluxes of an equivalent conservation law. From the practical point of view, for a given set of multipliers, it is sufficient to find any one set of corresponding fluxes.

We now consider different ways of finding fluxes of conservation laws from known multipliers.

\subsection{The direct method of flux computation}

For conservation laws arising from simple forms of multipliers, the fluxes are most easily found by direct matching of the two sides of equation (3.1) and subsequent integration by parts.

If integration by parts is not obvious, one can assume a sufficiently general dependence for the fluxes $\Phi^{i}[U]$, and look for a particular solution of PDEs (3.1) for the fluxes within such an ansatz. Here it is important to note that if the maximal order of derivatives of $U(x)$ present in the multipliers is $l$, and the maximal order in the equations $R^{\sigma}[U]$ appearing in the linear combination (3.1) is $k$, then, without loss of generality (i.e., up to trivial conservation laws) one may assume that the fluxes are of the form $\Phi^{i}[U]=\Phi^{i}\left(x, U, \partial U, \ldots, \partial^{r} U\right)$, where $r=\max (l, k)$ [23]. Using the 
independence of $\Phi^{i}[U]$ from higher derivatives, one can subsequently split PDEs (3.1) into a set of simpler determining equations.

We now consider an example of a direct flux computation. Further examples are found in Section 6 .

Example. As an example, consider the nonlinear wave equation

$$
R[u]=u_{t t}-\left(c^{2}(u) u_{x}\right)_{x}=0,
$$

for arbitrary wave speed $c(u)$. For simplicity, consider multipliers of the form $\Lambda[U]=\Lambda(x, t, U)$. The determining equations (3.3) yield the solution $\Lambda(x, t)=C_{1}+C_{2} x+C_{3} t+C_{4} x t$, where $C_{1}, \ldots, C_{4}$ are arbitrary constants. As a result, one obtains four linearly independent conservation laws, arising from the multipliers $\Lambda^{(1)}=1, \Lambda^{(2)}=x, \Lambda^{(3)}=t, \Lambda^{(4)}=x t$. We now determine the corresponding density-flux pairs.

For the multiplier $\Lambda^{(1)}=1$, one obviously has

$$
\Lambda^{(1)}[U] R[U] \equiv \mathrm{D}_{t}\left(U_{t}\right)-\mathrm{D}_{x}\left(c^{2}(U) U_{x}\right),
$$

since PDE (4.1) is in divergence form as it stands:

$$
\mathrm{D}_{t}\left(u_{t}\right)-\mathrm{D}_{x}\left(c^{2}(u) u_{x}\right)=0
$$

For the multiplier $\Lambda^{(2)}=x$, one can determine the flux and density using integration by parts:

$$
\begin{aligned}
\Lambda^{(2)}[U] R[U] & \equiv x\left(\mathrm{D}_{t}\left(U_{t}\right)-\mathrm{D}_{x}\left(c^{2}(U) U_{x}\right)\right) \\
& \equiv \mathrm{D}_{t}\left(x U_{t}\right)-\mathrm{D}_{x}\left(x c^{2}(U) U_{x}\right)+c^{2}(U) U_{x} \\
& \equiv \mathrm{D}_{t}\left(x U_{t}\right)-\mathrm{D}_{x}\left(x c^{2}(U) U_{x}-\int c^{2}(U) d U\right)
\end{aligned}
$$

and thus the corresponding conservation law is given by

$$
\mathrm{D}_{t}\left(x u_{t}\right)-\mathrm{D}_{x}\left(x c^{2}(u) u_{x}-\int c^{2}(u) d u\right)=0 .
$$

Similarly, for the multiplier $\Lambda^{(3)}=t$, one finds the corresponding conservation law

$$
\mathrm{D}_{t}\left(t u_{t}-u\right)-\mathrm{D}_{x}\left(t c^{2}(u) u_{x}\right)=0 .
$$

To find the flux and density of the somewhat more complicated fourth conservation law of PDE (4.1) following from the multiplier $\Lambda^{(4)}=x t$, one needs to solve the flux-density determining equation

$$
\Lambda^{(4)}[U] R[U]=x t\left(\mathrm{D}_{t}\left(U_{t}\right)-\mathrm{D}_{x}\left(c^{2}(U) U_{x}\right)\right) \equiv \mathrm{D}_{t} \Psi[U]+\mathrm{D}_{x} \Phi[U]
$$

Since the left-hand side of (4.7) is linear in the highest derivatives $U_{t t}$ and $U_{x x}$, one can assume that $\Psi[U]=\Psi\left(x, t, U, U_{x}, U_{t}\right)$ and $\Phi[U]=\Phi\left(x, t, U, U_{x}, U_{t}\right)$. Expanding both sides of (4.7), one obtains

$$
\begin{gathered}
x t\left(U_{t t}-2 c(U) c^{\prime}(U)\left(U_{x}\right)^{2}-c^{2}(U) U_{x x}\right) \\
\quad=\left(\Psi_{t}+\Psi_{U} U_{t}+\Psi_{U_{t}} U_{t t}+\Psi_{U_{x}} U_{x t}\right) \\
\quad+\left(\Phi_{x}+\Phi_{U} U_{x}+\Phi_{U_{t}} U_{x t}+\Phi_{U_{x}} U_{x x}\right) .
\end{gathered}
$$


Matching the terms of the highest order derivatives $U_{t t}, U_{x x}$ and $U_{x t}$, one finds that

$$
x t=\Psi_{U_{t}}, \quad-x t c^{2}(U)=\Phi_{U_{x}}, \quad \Psi_{U_{x}}=-\Phi_{U_{t}} .
$$

The third equation in (4.9) can be replaced by $\Psi_{U_{x}}=\Phi_{U_{t}}=0$, since other choices lead to equivalent conservation laws. The first two equations in (4.9) yield

$$
\Psi[U]=x t U_{t}+\alpha(x, t, U), \quad \Phi[U]=-x t c^{2}(U) U_{x}+\beta(x, t, U)
$$

for arbitrary $\alpha(x, t, U), \beta(x, t, U)$. Substituting (4.10) into the determining equations (4.8) and setting to zero coefficients of first-order partial derivatives of $U$, one finds

$$
x=-\alpha_{U}, \quad t c^{2}(U)=\beta_{U}, \quad \alpha_{t}=-\beta_{x} .
$$

Therefore

$$
\alpha(x, t, U)=-x U+\widetilde{\alpha}(x, t), \quad \beta(x, t, U)=t \int c^{2}(U) d U+\widetilde{\beta}(x, t), \quad \widetilde{\alpha}_{t}=-\widetilde{\beta}_{x} .
$$

It is evident that any choice of $\widetilde{\alpha}$ and $\widetilde{\beta}$ satisfying $\widetilde{\alpha}_{t}=-\widetilde{\beta}_{x}$ yields an equivalent conservation law, with the simplest one having $\widetilde{\alpha}=\widetilde{\beta}=0$. Thus, the fourth conservation law of PDE (4.1) is given by

$$
\mathrm{D}_{t}\left(x t u_{t}-x u\right)-\mathrm{D}_{x}\left(x t c^{2}(u) u_{x}-t \int c^{2}(u) d u\right)=0
$$

\subsection{The first homotopy formula}

In the case of complicated forms of multipliers and/or equations, for the inversion of divergence operators, one can use homotopy operators that arise in differential geometry and reduce the problem of finding fluxes to a problem of integration in single-variable calculus. In this section, we present the first set of such formulas, following [19].

Definition 9. The $n$-dimensional higher Euler operator with respect to a function $U\left(x^{1}, \ldots, x^{n}\right)$ is given by

$$
\mathrm{E}_{U}^{\left(s_{1}, \ldots, s_{n}\right)}=\sum_{k_{1}=s_{1}}^{\infty} \ldots \sum_{k_{n}=s_{n}}^{\infty}\left(\begin{array}{c}
k_{1} \\
s_{1}
\end{array}\right) \ldots\left(\begin{array}{c}
k_{n} \\
s_{n}
\end{array}\right) \mathrm{D}_{1}^{k_{1}-s_{1}} \ldots \mathrm{D}_{n}^{k_{n}-s_{n}} \frac{\partial}{\partial U^{\left(k_{1}+\ldots+k_{n}\right)}},
$$

where one denotes

$$
U^{\left(k_{1}+\ldots+k_{n}\right)}=\frac{\partial^{k_{1}+\ldots+k_{n}} U}{\partial^{k_{1}} x^{1} \ldots \partial^{k_{n}} x^{n}}
$$

Note that $\mathrm{E}_{U}^{(0,0)}=\mathrm{E}_{U}$ is the usual Euler operator (2.5) with respect to $U$.

Definition 10. Let $x=\left(x^{1}, \ldots, x^{n}\right)$ and $U=\left(U^{1}(x), \ldots, U^{m}(x)\right)$. For an expression $f[U]=$ $f(x, U, \partial U, \ldots)$, the $n$-dimensional homotopy operator is defined through its $n$ components corresponding to $n$ respective independent variables $x^{i}, i=1, \ldots, n$, as follows:

$$
\mathrm{H}^{\left(x^{i}\right)}(f[U])=\left.\int_{0}^{1} \sum_{j=1}^{m} \mathrm{I}_{j}^{\left(x^{i}\right)}(f[\widehat{U}])\right|_{\widehat{U}=\lambda U} \frac{d \lambda}{\lambda},
$$


with

$$
\begin{aligned}
\mathrm{I}_{j}^{\left(x^{i}\right)}(f[\widehat{U}])= & \sum_{s_{1}=0}^{\infty} \ldots \sum_{s_{n}=0}^{\infty}\left(\frac{1+s_{i}}{1+s_{1}+\ldots+s_{n}}\right) \\
& \times \mathrm{D}_{1}^{s_{1}} \ldots \mathrm{D}_{n}^{s_{n}}\left(\widehat{U}^{j} \mathrm{E}_{\widehat{U}^{j}}^{\left(s_{1}, \ldots, s_{i-1}, 1+s_{i}, s_{i+1}, \ldots, s_{n}\right)}(f[\widehat{U}])\right),
\end{aligned}
$$

$j=1, \ldots, m$.

The following theorem holds [19].

Theorem 4. Suppose $f[U]$ is a divergence expression

$$
f[U]=\operatorname{div} \boldsymbol{\Phi}[U]=\mathrm{D}_{1} \Phi^{1}[U]+\ldots+\mathrm{D}_{n} \Phi^{n}[U],
$$

and $f[0]=0$. Then the fluxes $\Phi^{i}[U]$ are given by

$$
\Phi^{i}[U]=\mathrm{H}^{\left(x^{i}\right)}(f[U]), \quad i=1, \ldots, n .
$$

up to corresponding fluxes of a trivial conservation law, provided that the integrals (4.13) converge.

Two examples of the use of the first homotopy formula are now considered. Further examples are found in Section 6 .

Example 1. As a first example, consider the Korteweg-de Vries (KdV) equation

$$
u_{t}+u u_{x}+u_{x x x}=0 .
$$

One can show that PDE (4.16) has a local conservation law arising from the multiplier $\Lambda[U]=U$, i.e.,

$$
f[U]=U\left(U_{t}+U U_{x}+U_{x x x}\right) \equiv \mathrm{D}_{t} \Psi[U]+\mathrm{D}_{x} \Phi[U] .
$$

is a divergence expression. We now reconstruct the corresponding density $\Psi[U]$ and flux $\Phi[U]$. First compute

$$
\begin{aligned}
I^{(t)}(f[\widehat{U}])= & \mathrm{D}_{t}^{0} \mathrm{D}_{x}^{0}\left(\widehat{U} \mathrm{E}_{\widehat{U}}^{(1,0)}(f[\widehat{U}])\right)=\widehat{U}^{2}, \\
I^{(x)}(f[\widehat{U}]) & =\mathrm{D}_{t}^{0} \mathrm{D}_{x}^{2}\left(\widehat{U} \mathrm{E}_{\widehat{U}}^{(0,3)}(f[\widehat{U}])\right)+\mathrm{D}_{t}^{0} \mathrm{D}_{x}^{1}\left(\widehat{U} \mathrm{E}_{\widehat{U}}^{(0,2)}(f[\widehat{U}])\right)+\mathrm{D}_{t}^{0} \mathrm{D}_{x}^{0}\left(\widehat{U} \mathrm{E}_{\widehat{U}}^{(0,1)}(f[\widehat{U}])\right) \\
& =\widehat{U}^{3}-\widehat{U}_{x}^{2}+2 \widehat{U} \widehat{U}_{x x} .
\end{aligned}
$$

Consequently,

$$
\begin{aligned}
& \mathrm{H}^{(t)}(f[U])=\int_{0}^{1}(\lambda U)^{2} \frac{d \lambda}{\lambda}=\frac{1}{2} U^{2} \\
& \mathrm{H}^{(x)}(f[U])=\int_{0}^{1}\left(\lambda^{3} U^{3}-\lambda^{2} U_{x}^{2}+2 \lambda^{2} U U_{x x}\right) \frac{d \lambda}{\lambda}=\frac{1}{3} U^{3}-\frac{1}{2} U_{x}^{2}+U U_{x x},
\end{aligned}
$$

The corresponding conservation law is given by

$$
\mathrm{D}_{t}\left(\frac{1}{2} u^{2}\right)+\mathrm{D}_{x}\left(\frac{1}{3} u^{3}-\frac{1}{2} u_{x}^{2}+u u_{x x}\right)=0 .
$$


Example 2. Consider the 2-dimensional G-equation

$$
R[g]=g_{t}-|\operatorname{grad} g| \equiv g_{t}-\sqrt{g_{x}^{2}+g_{y}^{2}}=0,
$$

which describes flame propagation in a static gas (see, e.g., [24]). Here $g(t, x, y)=0$ implicitly defines the position of the flame surface at time $t$, and the surface advances at a constant speed in the normal direction. Let $\left(x^{1}, x^{2}\right)=(x, y)$. In particular, one can show that the PDE (4.18) has the following multipliers of conservation laws:

$$
\Lambda^{(1)}[G]=\frac{1}{G_{y}^{3}}\left(G_{x} G_{y y}-G_{y} G_{x y}\right), \quad \Lambda^{(2)}[G]=H\left(G_{x}, G_{y}\right)\left(G_{x x} G_{y y}-G_{x y}^{2}\right),
$$

where $H\left(G_{x}, G_{y}\right)$ is an arbitrary function. For more details, see [25].

First consider the multiplier $\Lambda^{(1)}[G]$ in (4.19). From (4.14), one obtains

$$
\mathrm{I}^{(t)}\left(\Lambda^{(1)}[G] R[G]\right)=\frac{G}{G_{x}^{3}}\left(G_{x} G_{x y}-G_{y} G_{x x}\right),
$$

which is an invariant expression under the transformation $\widehat{U}=\lambda U$. The same is true about expressions $\mathrm{I}^{(x)}\left(\Lambda^{(1)}[G] R[G]\right)$ and $\mathrm{I}^{(y)}\left(\Lambda^{(1)}[G] R[G]\right)$. Hence the integral homotopy operators (4.13) yield a divergent integral $\int_{0}^{1} d \lambda / \lambda$.

Second, consider the multiplier $\Lambda^{(2)}[G]$ in (4.19). Since the corresponding divergence expression

$$
f[G]=\Lambda^{(2)}[G] R[G]=H\left(G_{x}, G_{y}\right)\left(G_{t}-\sqrt{G_{x}^{2}+G_{y}^{2}}\right)\left(G_{x x} G_{y y}-G_{x y}^{2}\right)
$$

contains an arbitrary function $H\left(G_{x}, G_{y}\right)$, the corresponding integrals (4.13) cannot yield closedform expressions.

It follows that the present flux construction method cannot be used for either of the multipliers (4.19) of the $G$-equation (4.18).

\subsection{The second homotopy formula}

An alternative set of integral formulas for fluxes of local conservation laws was developed by Bluman and Anco [17]. Here we present these formulas in a different form, which is symmetric with respect to independent variables, and also is simpler for practical computations and software implementation.

It takes a somewhat bigger effort to implement the current method in symbolic computation software than to implement the method presented in Section 4.2. However the formulas given in the current section have two important practical advantages over the ones presented in Section 4.2 .

(i) They can be applied to divergence expressions $f[U]$ with $f[0] \neq 0$.

(ii) They have flexibility that can be used to always avoid divergent integrals.

The following theorem holds.

Theorem 5. Suppose a PDE system (2.1) has a local conservation law arising from a set of multipliers $\left\{\Lambda_{\sigma}[U]\right\}_{\sigma=1}^{N}$, i.e., (3.2) holds. Then the fluxes $\left\{\Phi^{i}[U]\right\}_{i=1}^{N}$ of that conservation law are given by

$$
\begin{aligned}
\Phi^{i}[U]= & \Phi^{i}[\widetilde{U}]+\int_{0}^{1}\left(S^{i}[U-\widetilde{U}, \Lambda[\lambda U+(1-\lambda) \widetilde{U}] ; R[\lambda U+(1-\lambda) \widetilde{U}]]\right. \\
& \left.+\widetilde{S}^{i}[U-\widetilde{U}, R[\lambda U+(1-\lambda) \widetilde{U}] ; \Lambda[\lambda U+(1-\lambda) \widetilde{U}]]\right) d \lambda, \quad i=1, \ldots, n,
\end{aligned}
$$


up to fluxes of a trivial conservation law. In (4.20), operators $S^{i}, \widetilde{S}^{i}$ are defined by their action on arbitrary functions $V=\left(V^{1}(x), \ldots, V^{m}(x)\right), W=\left(W_{1}(x), \ldots, W_{N}(x)\right), \widetilde{V}=\left(\widetilde{V}^{1}(x), \ldots, \widetilde{V}^{m}(x)\right)$ and $\widetilde{W}=\left(\widetilde{W}_{1}(x), \ldots, \widetilde{W}_{N}(x)\right)$ as follows:

$$
\begin{aligned}
& S^{i}[V, W ; R[U]]=\sum_{p=0}^{k-1} \sum_{q=0}^{k-p-1}(-1)^{q}\left(\mathrm{D}_{i_{1}} \ldots \mathrm{D}_{i_{p}} V^{\rho}\right) \mathrm{D}_{j_{1}} \ldots \mathrm{D}_{j_{q}}\left(W_{\sigma} \frac{\partial R^{\sigma}[U]}{\partial U_{j_{1} \ldots j_{q} i i_{1} \ldots i_{p}}^{\rho}}\right) \\
& \widetilde{S}^{i}[\widetilde{V}, \widetilde{W} ; \Lambda[U]]=\sum_{p=0}^{l-1} \sum_{q=0}^{l-p-1}(-1)^{q}\left(\mathrm{D}_{i_{1}} \ldots \mathrm{D}_{i_{p}} \widetilde{V}^{\rho}\right) \mathrm{D}_{j_{1}} \ldots \mathrm{D}_{j_{q}}\left(\widetilde{W}^{\sigma} \frac{\partial \Lambda_{\sigma}[U]}{\partial U_{j_{1} \ldots j_{q} i i_{1} \ldots i_{p}}^{\rho}}\right),
\end{aligned}
$$

where $k$ is the order of the given PDE system $\mathbf{R}\{x ; u\}(2.1), l$ is the maximal order of the derivatives appearing in the multipliers $\left\{\Lambda_{\sigma}[U]\right\}_{\sigma=1}^{N}$, and $j_{1} \ldots j_{q}, i_{1} \ldots i_{p}$ are ordered combinations of indices such that $1 \leq j_{1} \leq \ldots \leq j_{q} \leq i \leq i_{1} \leq \ldots \leq i_{p} \leq n$.

In (4.20), $\widetilde{U}=\widetilde{U}(x)$ is an arbitrary fixed function, and $\Phi^{i}[\widetilde{U}]$ are quantities satisfying

$$
\mathrm{D}_{i} \Phi^{i}[\tilde{U}]=\Lambda_{\sigma}[\widetilde{U}] R^{\sigma}[\widetilde{U}]=: F(x) .
$$

The proof of Theorem 5 is presented in Appendix $\mathrm{A}$.

In (4.20), $\widetilde{U}$ is an arbitrary function of $x$, chosen so that the integral (4.20) converges. Different choices of $\widetilde{U}$ yield fluxes of equivalent conservation laws. One normally chooses $\widetilde{U}=0$ (provided that the integral converges). Once $\widetilde{U}=\widetilde{U}(x)$ has been chosen, the functions $\left\{\Phi^{i}[\widetilde{U}]\right\}_{i=1}^{N}$ can be found by direct integration from the divergence relation (4.23) by integrating $F(x)$. For example, one can choose

$$
\Phi^{1}[\widetilde{U}]=\int F(x) d x^{1}, \quad \Phi^{2}[\widetilde{U}]=\ldots=\Phi^{n}[\widetilde{U}]=0 .
$$

It is important that unlike the case with the first homotopy formula (Section 4.2), the proof of Theorem 5 does not require that the divergence expression $\Lambda_{\sigma}[U] R^{\sigma}[U]$ vanishes for $U=0$.

The formula (4.20) is useful in many practical situations when the integrals can be explicitly evaluated.

Two examples of the use of the second homotopy formula are now considered; further examples are found in Section 6 .

Example 1. As a first example, consider again the $\mathrm{KdV}$ equation (4.16) with multiplier $\Lambda[U]=U$. Using $\widetilde{U}=0$, one finds $\Phi^{1}[0]=\Phi^{2}[0]=0$, and hence the formulas (4.20) yield exactly the same fluxes as the first homotopy method (Section 4.2), i.e., the conservation law (4.17).

Alternatively, using $\widetilde{U}=x$, one finds $\Phi^{1}[\widetilde{U}]=t x^{2}, \Phi^{2}[\widetilde{U}]=0$, and hence

$$
\begin{aligned}
& \Phi^{1}[U]=t x^{2}+\frac{1}{2}\left(U^{2}-x^{2}\right), \\
& \Phi^{2}[U]=\frac{1}{2}-\frac{1}{3} x^{3}+\frac{1}{3} U^{3}-\frac{1}{2} U_{x}^{2}+U U_{x x},
\end{aligned}
$$

which are a density and a flux of a conservation law equivalent to (4.17).

Example 2. Consider the 2-dimensional $G$-equation (4.18) and its local conservation law multipliers (4.19). For the multiplier $\Lambda^{(1)}[G]$, it again yields a divergent integral when $\widetilde{U}=0$. The singularity can be removed by taking e.g. $\widetilde{U}=x$, however even this simple choice leads to an integral with a highly complicated integrand. For the multiplier $\Lambda^{(2)}[G]$ involving an arbitrary function, formulas (4.20) do not yield a closed-form expression. 


\subsection{Computation of fluxes of conservation laws of scaling-invariant PDE sys- tems}

Consider a PDE system that has a scaling symmetry

$$
\mathrm{X}_{s}[u]=p^{(i)} x^{i} \frac{\partial}{\partial x^{i}}+q^{(\rho)} u^{\rho} \frac{\partial}{\partial u^{\rho}},
$$

where $p^{(i)}$ and $q^{(\rho)}$ are constant scaling weights of independent and dependent variables, respectively, i.e., the generator (4.24) corresponds to the global transformation group

$$
\begin{aligned}
& x^{i} \rightarrow \tilde{x}^{i}=e^{\varepsilon \mathrm{X}_{s}[u]} x^{i}=e^{\varepsilon p^{(i)}} x^{i}, \\
& u^{\rho} \rightarrow \tilde{u}^{\rho}=e^{\varepsilon \mathrm{X}_{s}[u]} u^{\rho}=e^{\varepsilon q^{(\rho)}} u^{\rho} .
\end{aligned}
$$

The scaling symmetry generator (4.24) can be written in evolutionary form as

$$
\hat{\mathrm{X}}_{s}[u]=\hat{\eta}[u] \frac{\partial}{\partial u^{\rho}}=\left(q^{(\rho)} u^{\rho}-p^{(i)} x^{i} u_{i}^{\rho}\right) \frac{\partial}{\partial u^{\rho}} .
$$

Assuming that the given PDE system can be written in a solved form (2.2), we first note that the scaling homogeneity of each PDE of the given system follows from considering the action of the scaling symmetry (4.24) on the leading derivative in each $R^{\sigma}[u]$. In particular, one has

$$
\mathrm{X}_{s}^{(k)}[U] R^{\sigma}[U]=r^{(\sigma)} R^{\sigma}[U],
$$

where $r^{(\sigma)}=$ const is the scaling weight of each $\mathrm{PDE} R^{\sigma}[U]$.

Now suppose the given PDE system (2.2) has a conservation law

$$
\Lambda_{\sigma}[u] R^{\sigma}[u]=\mathrm{D}_{i} \Phi^{i}[u]=0
$$

with fluxes $\Phi^{i}[U]=\Phi^{i}\left(x, U, \partial U, \ldots, \partial^{l} U\right)$. Suppose that this conservation law is scaling-invariant, and moreover, homogeneous under the scaling symmetry (4.24), i.e.,

$$
\mathrm{X}_{s}^{(l)}[U] \mathrm{D}_{i} \Phi^{i}[U]=P \mathrm{D}_{i} \Phi^{i}[U]
$$

where $\mathrm{X}_{s}^{(l)}$ is a corresponding prolongation of the scaling symmetry generator (4.24), and $P=$ const is a scaling weight of the conservation law.

Then one can show [21] that each of the multipliers $\Lambda_{\sigma}[U]$ appearing in (4.26) is homogeneous under the scaling symmetry (4.24). In particular, $\mathrm{X}_{s}^{(l)}[U] \Lambda_{\sigma}[U]=s_{(\sigma)} \Lambda_{\sigma}[U]$, where $s_{(\sigma)}=P-r^{(\sigma)}$ is the scaling weight of each $\Lambda_{\sigma}[U] \neq 0$.

Definition 11. A conservation law (4.26) invariant with respect to a scaling symmetry $\mathrm{X}_{s}[u]$ (4.24) is called noncritical with respect to $\mathrm{X}_{s}[u]$ if

$$
\chi=s_{(\sigma)}+r^{(\sigma)}+\sum_{i=1}^{n} p^{(i)} \neq 0
$$

for each $\sigma$ such that $\Lambda_{\sigma}[U] \neq 0$. 


\section{The formula for fluxes}

It follows that fluxes of noncritical homogeneously scaling conservation laws of scaling-invariant PDE systems can be found through a simple formula that involves no integration! The following theorem holds $[20,21]$.

Theorem 6. Suppose the PDE system in the solved form (2.2) has a scaling symmetry (4.25), and a conservation law (2.6), which is homogeneous under the scaling symmetry (4.24), and is noncritical with respect to it. Then the fluxes $\Phi^{i}[U]$ of such conservation law are given by

$$
\Phi^{i}[U]=\sum_{p=0}^{k-1} \sum_{q=0}^{k-p-1}(-1)^{q}\left(\mathrm{D}_{i_{1}} \ldots \mathrm{D}_{i_{p}} \hat{\eta}^{\rho}\right) \mathrm{D}_{j_{1}} \ldots \mathrm{D}_{j_{q}}\left(\Lambda_{\sigma}[U] \frac{\partial R^{\sigma}[U]}{\partial U_{j_{1} \ldots j_{q} i i_{1} \ldots i_{p}}^{\rho}}\right)
$$

up to fluxes of a trivial conservation law. In (4.29), $k$ is the maximal order of derivatives appearing in the PDE system (2.1), $l$ is the maximal order of derivatives appearing in the multipliers $\left\{\Lambda_{\sigma}[U]\right\}_{\sigma=1}^{N}$, and $j_{1} \ldots j_{q}$ and $i_{1} \ldots i_{p}$ are ordered combinations of indices such that $1 \leq j_{1} \leq \ldots \leq$ $j_{q} \leq i \leq i_{1} \leq \ldots \leq i_{p} \leq n$.

Remark 1. From the proof of Theorem 6 it follows that the formulas (4.29) does not yield fluxes of critical conservation laws. In particular, for critical conservation laws, formulas (4.29) yield fluxes of trivial conservation laws.

In practice, if a PDE system has two or more scaling symmetries, it can be the case that a particular conservation law is critical with respect to one scaling and noncritical with respect to the other, as is the case in the Example 2 below.

Remark 2. For first- and second-order PDE systems, the flux formula (4.29) takes a particularly simple form [20]. For second-order PDE systems, it becomes

$$
\Phi^{i}[U]=\hat{\eta}^{\rho}[U] \Lambda_{\sigma}[U] \frac{\partial R^{\sigma}[U]}{\partial u_{i}^{\rho}}+\left(\mathrm{D}_{s} \hat{\eta}^{\rho}[U]\right) \Lambda_{\sigma}[U] \frac{\partial R^{\sigma}[U]}{\partial u_{i s}^{\rho}}-\hat{\eta}^{\rho}[U] \mathrm{D}_{j}\left(\Lambda_{\sigma}[U] \frac{\partial R^{\sigma}[U]}{\partial u_{j i}^{\rho}}\right),
$$

where the summation is taken over repeated indices satisfying $s \geq i$ and $j \leq i$. For first-order PDE systems, it further reduces to

$$
\Phi^{i}[U]=\hat{\eta}^{\rho}[U] \Lambda_{\sigma}[U] \frac{\partial R^{\sigma}[U]}{\partial u_{i}^{\rho}} .
$$

Remark 3. In general, one can obtain fluxes of conservation laws using formula (4.29) not only for scaling symmetries, but for any pair consisting of a local symmetry $\hat{\mathrm{X}}_{s}[u]=\hat{\eta}[u] \frac{\partial}{\partial u^{\rho}}$ (in the evolutionary form) and a set of local conservation law multipliers $\left\{\Lambda_{\sigma}[U]\right\}_{\sigma=1}^{N}$. Details are given in Appendix B (see also $[16,21])$.

Example 1. As a first example, we consider again the KdV equation (4.16) with multiplier $\Lambda[U]=U$. The PDE (4.16) has a scaling symmetry

$$
\mathrm{X}_{s}[u]=x \frac{\partial}{\partial x}+3 t \frac{\partial}{\partial t}-2 u \frac{\partial}{\partial u} .
$$


Using the formula (4.29) with $\left(x^{1}, x^{2}\right)=(t, x)$, one obtains the density and the flux

$$
\begin{aligned}
\Phi^{1}[U]= & \left(2 U+3 t U_{t}+x U_{x}\right) U \\
\Phi^{2}[U]= & U\left(3 t U_{t x x}+x U_{x x x}\right)-3 t U_{x} U_{t x} \\
& +3\left(2 U+t U_{t}\right) U_{x} x+U^{2}\left(2 U+3 t U_{t}\right)+U_{x}\left(x U^{2}-3 U_{x}\right),
\end{aligned}
$$

i.e., a conservation law

$$
\begin{aligned}
\mathrm{D}_{t}( & \left.\left(2 u+3 t u_{t}+x u_{x}\right) u\right)+\mathrm{D}_{x}\left(u\left(3 t u_{t x x}+x u_{x x x}\right)-3 t u_{x} u_{t x}\right. \\
& \left.+3\left(2 u+t u_{t}\right) u_{x} x+u^{2}\left(2 u+3 t u_{t}\right)+u_{x}\left(x u^{2}-3 u_{x}\right)\right)=0
\end{aligned}
$$

equivalent to the conservation law (4.17).

Example 2. Consider the 2-dimensional $G$-equation (4.18) and its local conservation law multipliers (4.19). The $G$-equation (4.18) is a first-order PDE with obvious scaling symmetries

$$
\mathrm{X}_{1}=g \frac{\partial}{\partial g}, \quad \mathrm{X}_{2}=t \frac{\partial}{\partial t}+x \frac{\partial}{\partial x}+y \frac{\partial}{\partial y} .
$$

Denoting $\left(x^{1}, x^{2}, x^{3}\right) \equiv(t, x, y)$, for $\mathrm{X}_{1}$, one has $q=1, p^{(1)}=p^{(2)}=p^{(3)}=0$; for $\mathrm{X}_{2}, q=0$, $p^{(1)}=p^{(2)}=p^{(3)}=1$.

Consider the conservation of the $G$-equation (4.18) law with multiplier $\Lambda^{(1)}[G]=\frac{1}{G_{y}^{3}}\left(G_{x} G_{y y}-\right.$ $G_{y} G_{x y}$ ) (4.19). The function $\Lambda^{(1)}[G]$ is evidently homogeneous under both symmetries $\mathrm{X}_{1}$ and $\mathrm{X}_{2}$ (4.34), with respective scaling weights $s_{1}=-1$ and $s_{2}=0$. The corresponding scaling weights of the $G$-equation (4.18) are $r_{1}=1$ and $r_{2}=-1$.

Using Definition 11, one finds $\chi_{1}=-1+1=0$, and $\chi_{2}=0-1+3=2$, hence the conservation law with multiplier $\Lambda^{(1)}[G]$ is critical with respect to the scaling symmetry $\mathrm{X}_{1}$ and noncritical with respect to $\mathrm{X}_{2}$. Indeed, one can directly check that the conservation law obtained by formulas (4.29) using the symmetry $\mathrm{X}_{1}$,

$$
\mathrm{D}_{t}\left(g \Lambda^{(1)}[g]\right)-\mathrm{D}_{x}\left(\frac{g g_{x} \Lambda^{(1)}[g]}{\sqrt{g_{x}^{2}+g_{y}^{2}}}\right)-\mathrm{D}_{y}\left(\frac{g g_{y} \Lambda^{(1)}[g]}{\sqrt{g_{x}^{2}+g_{y}^{2}}}\right)=0,
$$

is trivial. The correct conservation law fluxes corresponding to the multiplier $\Lambda^{(1)}[G]$ can be found using the symmetry $\mathrm{X}_{2}$; such conservation law is given by

$$
\begin{aligned}
& \mathrm{D}_{t}\left(\hat{\eta}[g] \Lambda^{(1)}[g]\right)-\mathrm{D}_{x}\left(\frac{\hat{\eta}[g] g_{x} \Lambda^{(1)}[g]}{\sqrt{g_{x}^{2}+g_{y}^{2}}}\right)-\mathrm{D}_{y}\left(\frac{\hat{\eta}[g] g_{y} \Lambda^{(1)}[g]}{\sqrt{g_{x}^{2}+g_{y}^{2}}}\right)=0, \\
& \hat{\eta}[g]=-t g_{t}-x g_{x}-y g_{y} .
\end{aligned}
$$

\section{Comparison of the four flux computation methods}

In Table 1, we briefly outline the characteristics of the four methods of flux computation discussed above. 
Table 1: Comparison of Four Methods of Flux Computation of Section 4.

\begin{tabular}{|l|l|l|}
\hline \multicolumn{1}{|c|}{ Method } & \multicolumn{1}{|c|}{ Applicability } & Computational complexity \\
\hline \hline $\begin{array}{l}\text { Direct } \\
\text { (Section 4.1) }\end{array}$ & $\begin{array}{l}\text { Simpler multipliers/PDE systems, which may in- } \\
\text { volve arbitrary functions. }\end{array}$ & $\begin{array}{l}\text { Solution of an overdeter- } \\
\text { mined linear PDE system } \\
\text { for fluxes. }\end{array}$ \\
\hline $\begin{array}{l}\text { Homotopy 1 } \\
\text { (Section 4.2) }\end{array}$ & $\begin{array}{l}\text { Complicated multipliers/PDEs, not involving arbi- } \\
\text { trary functions. } \\
\text { The divergence expression must vanish for } U=0 . \\
\text { For some conservation laws, this method can yield } \\
\text { tion. } \\
\text { divergent integrals. }\end{array}$ \\
\hline $\begin{array}{l}\text { Homotopy 2 } \\
\text { (Section 4.3) }\end{array}$ & $\begin{array}{l}\text { Complicated multipliers/PDEs, not involving arbi- } \\
\text { trary functions. }\end{array}$ & $\begin{array}{l}\text { One-dimensional integra- } \\
\text { tion. }\end{array}$ \\
\hline $\begin{array}{l}\text { Scaling sym- } \\
\text { metry }\end{array}$ & $\begin{array}{l}\text { Complicated multipliers/PDEs, may involve arbi- } \\
\text { trary functions. }\end{array}$ & Repeated differentiation. \\
Section 4.4) & $\begin{array}{l}\text { Scaling-homogeneous PDEs and multipliers. } \\
\text { Noncritical conservation laws. }\end{array}$ & \\
\hline
\end{tabular}

One can see that there is no "preferred" method of finding fluxes that is simple to use and sufficiently general to be recommended for a generic conservation law of a nonlinear PDE system. The following observations and recommendations are based on the author's experience.

- If a given PDE system and multipliers do not involve arbitrary functions, one usually uses one of the two integral methods. Though the second integral method is more general, the first one is also sometimes used because it can yield simpler flux expressions.

- If a given PDE system and/or multipliers are not very complicated but involve arbitrary constitutive functions, one normally attempts the direct method of flux computation.

- When a given PDE system is scaling-invariant and the conservation law is scaling-homogeneous, then the scaling symmetry method is the method of choice, since it involves simplest computations. (However, as seen in the KdV example (4.33), when some $p^{(i)} \neq 0$, the densities and fluxes computed through the fourth method can contain multiple unnecessary terms corresponding to trivial conservation laws.)

- Another instance when one needs to use the fourth method is the case of complicated scalinghomogeneous PDEs and/or multipliers involving arbitrary functions. In this situation, the scaling symmetry method is the only systematic method available.

In the following Section 5, we consider a software implementation of the four above-discussed flux computation methods, applied to several practical examples. 


\section{Symbolic software implementation of flux computation meth- ods. Examples}

The direct method of construction of conservation laws (Section 3.1) is algorithmic and has been

implemented in symbolic softwarea). Here we discuss its Maple implementation in the symbolic package GeM [22] written by the author. The package GeM also contains routines for the four methods of flux reconstruction considered in this paper.

\subsection{General conservation laws of the nonlinear wave equation}

Here we seek local conservation laws for the nonlinear wave equation (4.1) arising from the multipliers of the form $\Lambda[U]=\Lambda(x, t, U)$ for an arbitrary $c(u)$.

One defines the variables and the PDE (4.1) in GeM using the following Maple commands:

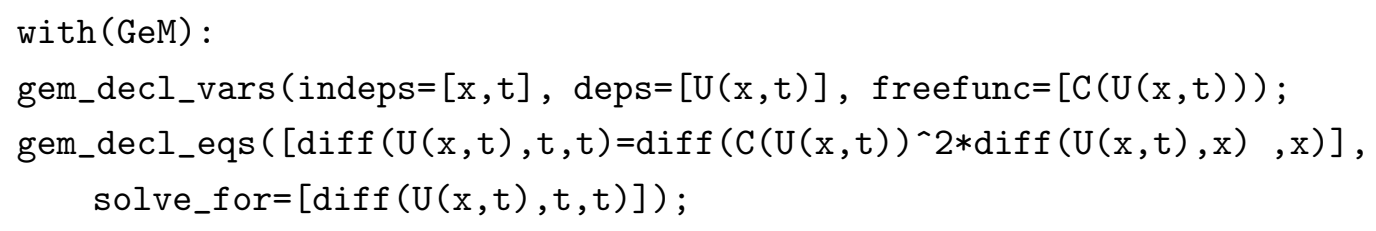

The option solve_for is used to specify a set of leading derivatives the given PDE systems can be solved for. Note that the direct method of conservation law computation does not require the equations to be written in the solved form, and therefore the specification of the option solve_for is not necessary. However in (6.1), this option solve_for was specified, since it will be used later in the flux computation routine, which automatically verifies the correctness of flux computations, which is accomplished by explicitly checking that the conservation law divergence expression vanishes on solutions $U(x)=u(x)$ of the given PDE (4.1).

The set of determining equations for the local conservation law multipliers is obtained and simplified using the routines

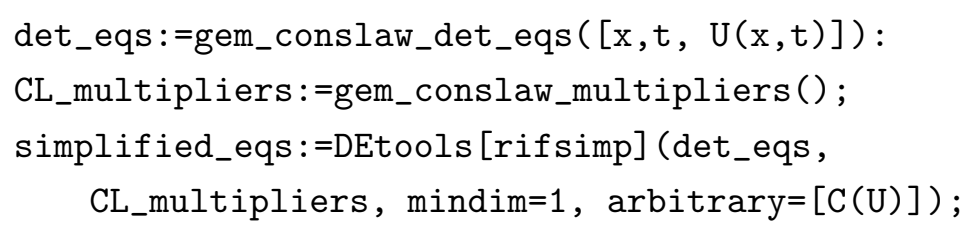

The first command in (6.2) sets up the set of local conservation law multipliers determining equations (3.4), and splits them. The splitting is done using the fact that the determining equations (3.4) are polynomial expressions in terms of derivatives $U$ of orders four and higher, and such derivatives are linearly independent. This yields an overdetermined system of 7 determining equations for the unknown local multiplier $\Lambda[U]$. After using the rif reduction algorithm, the system reduces to three PDEs

$$
\frac{\partial^{2} \Lambda[U]}{\partial x^{2}}=\frac{\partial^{2} \Lambda[U]}{\partial t^{2}}=\frac{\partial \Lambda[U]}{\partial U}=0
$$

The four obvious linearly independent solutions of these determining equations can be obtained using the command

multipliers_sol:=pdsolve(simplified_eqs [Solved]);

\footnotetext{
a) In particular, in the software by T. Wolf [26] written for REDUCE computer algebra system.
} 
and are given by

$$
\Lambda[U]=C_{1}+C_{2} x+C_{3} t+C_{4} t x
$$

where $C_{1}, \ldots, C_{4}$ are arbitrary constants.

Finally, we apply the direct method of flux computation (Section 4.1), using the command

$$
\text { gem_get_CL_fluxes (Lam_sol, method="Direct") ; }
$$

which generates the divergence expressions of the four conservation laws (4.3), (4.5), (4.6) and (4.11).

\subsection{The first four conservation laws of the $\mathrm{KdV}$ equation}

As it is well-known, the KdV equation (4.16) has an infinite countable sequence of local conservation laws of increasing orders. Here we compute the first four of these conservation laws, by limiting the multiplier dependence, as follows:

$$
\Lambda[U]=\Lambda\left(t, x, U, U_{x}, U_{x x}\right)
$$

The variables and the PDE (4.16) are defined using the commands

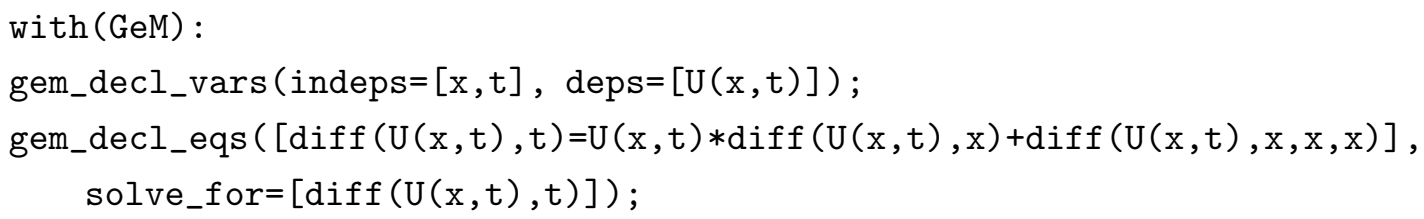

The set of determining equations for the multipliers is obtained and simplified using the routines

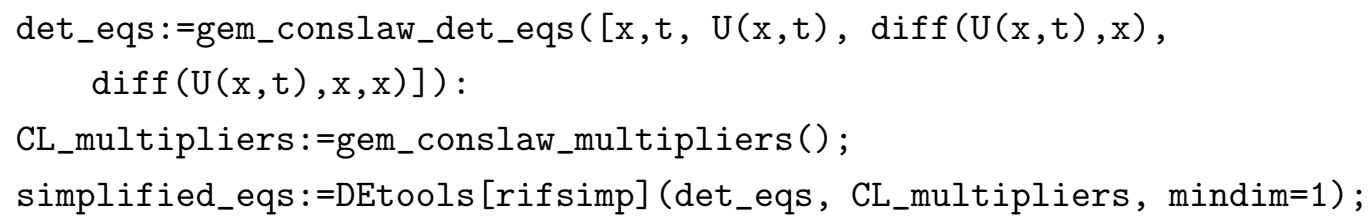

and is solved using the Maple command

multipliers_sol:=pdsolve(simplified_eqs [Solved]) ;

to yield the four local multipliers

$$
\Lambda[U]=C_{1}+C_{2} U+C_{3}(x-t U)+C_{4}\left(\frac{1}{2} U^{2}+U_{x x}\right),
$$

where $C_{1}, \ldots, C_{4}$ are arbitrary constants.

We now reconstruct the fluxes.

1. The first homotopy formula. Using the command

$$
\text { gem_get_CL_fluxes (Lam_sol, method="Homotopy1"); }
$$


we call the routine for the first homotopy method described in Section 4.2. It yields the conservation laws

$$
\begin{aligned}
& \mathrm{D}_{t}(u)+\mathrm{D}_{x}\left(\frac{1}{2} u^{2}+u_{x x}\right)=0 \\
& \mathrm{D}_{t}\left(\frac{1}{2} u^{2}\right)+\mathrm{D}_{x}\left(\frac{1}{3} u^{3}-\frac{1}{2} u_{x}^{2}+u u_{x x}\right)=0 \\
& \mathrm{D}_{t}\left(x u-\frac{1}{2} t u^{2}\right)+\mathrm{D}_{x}\left(-\frac{1}{3} t u^{3}+\frac{1}{2}\left(x u^{2}+t u_{x}^{2}\right)-u_{x}+(x-t u) u_{x x}\right)=0 \\
& \mathrm{D}_{t}\left(\frac{1}{6} u^{3}+\frac{1}{2} u u_{x x}\right)+\mathrm{D}_{x}\left(\frac{1}{8} u^{4}+\frac{1}{2}\left(u_{t} u_{x}-u u_{t x}+u^{2} u_{x x}+u_{x x}^{2}\right)\right)=0 .
\end{aligned}
$$

2. The second homotopy formula. The corresponding routine for the method described in Section 4.3 is called as follows

$$
\text { gem_get_CL_fluxes (Lam_sol, method="Homotopy2"); }
$$

and yields the four divergence expressions in the same form as in (6.7). By default, the arbitrary function $\widetilde{U}=0$ is used. One can override this setting and use, e.g., $\widetilde{U}=x$, by using an option, as follows:

$$
\text { gem_get_CL_fluxes (Lam_sol, method="Homotopy2", arb_func_Homotopy2=\{U=x }\} \text { ); }
$$

which yield four conservation laws equivalent to the ones given in (6.7), but having somewhat more complicated fluxes:

$$
\begin{aligned}
& \mathrm{D}_{t}(x(t-1)+u)+\mathrm{D}_{x}\left(\frac{1}{2}\left(u^{2}-x^{2}\right)+u_{x x}\right)=0, \\
& \mathrm{D}_{t}\left(t x^{2}+\frac{1}{2}\left(u^{2}-x^{2}\right)\right)+\mathrm{D}_{x}\left(\frac{1}{2}-\frac{1}{3} x^{3}+\frac{1}{3} u^{3}-\frac{1}{2} u_{x}^{2}+u u_{x x}\right)=0, \\
& \mathrm{D}_{t}\left(\frac{1}{2} x^{2}\left(3 t-t^{2}-2\right)+x u-\frac{1}{2} t u^{2}\right) \\
& \quad \quad+\mathrm{D}_{x}\left(1+t\left(\frac{1}{3} x^{3}-\frac{1}{2}\right)-\frac{1}{2} x^{2}-\frac{1}{3} t u^{3}+\frac{1}{2}\left(x u^{2}+t u_{x}^{2}\right)-u_{x}+(x-t u) u_{x x}\right)=0, \\
& \quad \mathrm{D}_{t}\left(\frac{1}{6} x^{3}(3 t-1)+\frac{1}{6} u^{3}+\frac{1}{2}(u-x) u_{x x}\right) \\
& \quad+\mathrm{D}_{x}\left(\frac{1}{8}\left(u^{4}-x^{4}\right)+\frac{1}{2}\left(u_{t}\left(u_{x}-1\right)-(u-x) u_{t x}+u^{2} u_{x x}+u_{x x}^{2}\right)\right)=0 .
\end{aligned}
$$

The above example illustrates that normally, to obtain simpler flux expressions, one should choose $\widetilde{U}=0$, unless integrals in the formula (4.20) diverge.

3. The scaling symmetry formula. We now reconstruct the conservation laws of the $\mathrm{KdV}$ equation (4.16) arising from local multipliers (6.5) using the scaling symmetry method (Section 4.4) with the scaling symmetry (4.32). In the corresponding routine, we need to specify the point symmetry (4.32) in the standard form:

$$
\begin{array}{r}
\text { gem_get_CL_fluxes (Lam_sol, method="Scaling", } \\
\text { symmetry } \left.=\left\{x i_{-} x=x, x i_{-} t=3 * t, \text { eta_U }=-2 * U\right\}\right) ;
\end{array}
$$


This yields the following four conservation law expressions

$$
\begin{aligned}
& \mathrm{D}_{t}\left(2 u+3 t u_{t}+x u_{x}\right)+\mathrm{D}_{x}\left(2 u^{2}+3 t u u_{t}+x u u_{x}+4 u_{x x}+3 t u_{t x x}+x u_{x x x}\right)=0, \\
& \mathrm{D}_{t}\left(\left(2 u+3 t u_{t}+x u_{x}\right) u\right)+\mathrm{D}_{x}\left(u\left(3 t u_{t x x}+x u_{x x x}\right)-3 t u_{x} u_{t x}\right. \\
& \left.\quad+3\left(2 u+t u_{t}\right) u_{x} x+u^{2}\left(2 u+3 t u_{t}\right)+u_{x}\left(x u^{2}-3 u_{x}\right)\right)=0, \\
& \mathrm{D}_{t}\left(\left(2 u+3 t u_{t}+x u_{x}\right)(x-t u)\right) \\
& \quad+\mathrm{D}_{x}\left(3 t u_{x}^{2}+2 u^{2}(x-t u)+[x u(x-t u)-3] u_{x}+3 t u(x-t u) u_{t}+3 t\left(t u_{x}-1\right) u_{t x}\right. \\
& \quad-3\left(2 u t+t^{2} u t-x\right) u_{x x}+(x-u t)\left(x u_{x x x}+3 t u_{t x x}\right)=0, \\
& \quad \mathrm{D}_{t}\left(\left(2 u+3 t u_{t}+x u_{x}\right)\left(\frac{1}{2} u^{2}+u_{x x}\right)\right) \\
& \quad+\mathrm{D}_{x}\left(\frac{1}{2} u^{3}\left(x u_{x}+3 t u_{t}\right)+u_{x}^{2}\left(3 t u_{t}+x u_{x}-u\right)+u^{4}-3 t u u_{x} u_{t x}\right. \\
& \quad+u\left[x u_{x}+6\left(t u_{t}+u\right)\right] u_{x x}+4 u_{x x}^{2}+\frac{3}{2} t\left(u^{2}+2 u_{x x}\right) u_{t x x} \\
& \left.\quad+\left[\frac{1}{2} x u^{2}-3\left(t u_{t x}+u_{x}\right)\right] u_{x x x}+\left(3 t u_{t}+x u_{x}+2 u\right) u_{x x x x}\right)=0,
\end{aligned}
$$

equivalent to the sets of conservation laws (6.7) and (6.9) but having significantly more complicated forms of fluxes and densities.

\section{Conclusion}

The direct method of construction of conservation laws is a systematic procedure applicable to all PDEs and PDE systems involving sufficiently smooth functions. The direct method builds on ideas present in the famous Noether's Theorem, in particular, the use of Euler differential operators, which identically annihilate any divergence expression. However, unlike Noether's Theorem, the direct method is not limited to PDE systems arising from some variational principle (i.e., self-adjoint PDE systems).

Within the the direct method, one seeks multipliers, such that a linear combination of the PDEs of the system system taken with these multipliers yields a divergence expression. Importantly, for PDE systems that can be written in a solved form (2.2), the direct method is complete, since for such systems, all conservation laws arise as linear combinations of equations of the system.

Determining equations for unknown multipliers are obtained from the action of Euler differential operators on a linear combination of the PDEs of a given system, and are in many ways similar to local symmetry determining equations.

We note that the direct method can be applied without modification to ODEs and ODE systems, yielding their integrating factors and first integrals.

After the multipliers are computed, fluxes of the corresponding divergence expression can be reconstructed using different approaches. Each approach can be better in one particular situation and worse or non-applicable in another (Section 5).

The algorithms for the direct method and the flux reconstruction have been implemented in the symbolic package GeM for Maple, which was used to illustrate the four methods presented in the present paper.

\section{Acknowledgements}


The author is thankful to George Bluman and Stephen Anco for multiple discussions, to anonymous referees for useful suggestions, and to the University of Saskatchewan and the National Sciences and Engineering Research Council of Canada for research support.

\section{A Proof of Theorem 5}

Proof. We start with a set of local conservation law multipliers $\left\{\Lambda_{\sigma}[U]\right\}_{\sigma=1}^{N}$ of a given PDE system (2.1). These multipliers satisfy (3.1).

The linearizing operator associated with the PDE system (2.1) is given by

$$
\begin{aligned}
& \left(\mathrm{L}_{R}\right)_{\rho}^{\sigma}[U] V^{\rho}=\left[\frac{\partial R^{\sigma}[U]}{\partial U^{\rho}}+\frac{\partial R^{\sigma}[U]}{\partial U_{i}^{\rho}} \mathrm{D}_{i}+\ldots+\frac{\partial R^{\sigma}[U]}{\partial U_{i_{1} \ldots i_{k}}^{\rho}} \mathrm{D}_{i_{1}} \ldots \mathrm{D}_{i_{k}}\right] V^{\rho}, \\
& \sigma=1, \ldots, N
\end{aligned}
$$

and its adjoint by

$$
\begin{aligned}
& \left(\mathrm{L}_{R}^{*}\right)_{\rho}^{\sigma}[U] W_{\sigma}=\frac{\partial R^{\sigma}[U]}{\partial U^{\rho}} W_{\sigma}-\mathrm{D}_{i}\left(\frac{\partial R^{\sigma}[U]}{\partial U_{i}^{\rho}} W_{\sigma}\right) \\
& +\ldots+(-1)^{k} \mathrm{D}_{i_{1}} \ldots \mathrm{D}_{i_{k}}\left(\frac{\partial R^{\sigma}[U]}{\partial U_{i_{1} \ldots i_{k}}^{\rho}} W_{\sigma}\right), \quad \rho=1, \ldots, m
\end{aligned}
$$

acting respectively on arbitrary functions $V=\left(V^{1}(x), \ldots, V^{m}(x)\right)$ and $W=\left(W_{1}(x), \ldots, W_{N}(x)\right)$.

For each multiplier $\Lambda_{\sigma}[U]=\Lambda_{\sigma}\left(x, U, \partial U, \ldots, \partial^{l} U\right)$, introduce the corresponding linearizing operator

$$
\begin{aligned}
& \left(\mathrm{L}_{\Lambda}\right)_{\sigma \rho}[U] \tilde{V}^{\rho}=\left[\frac{\partial \Lambda_{\sigma}[U]}{\partial U^{\rho}}+\frac{\partial \Lambda_{\sigma}[U]}{\partial U_{i}^{\rho}} \mathrm{D}_{i}+\ldots+\frac{\partial \Lambda_{\sigma}[U]}{\partial U_{i_{1} \ldots i_{l}}^{\rho}} \mathrm{D}_{i_{1}} \ldots \mathrm{D}_{i_{l}}\right] \widetilde{V}^{\rho}, \\
& \sigma=1, \ldots, N
\end{aligned}
$$

and its adjoint

$$
\begin{aligned}
& \left(\mathrm{L}_{\Lambda}^{*}\right)_{\sigma \rho}[U] \widetilde{W^{\sigma}}=\frac{\partial \Lambda_{\sigma}[U]}{\partial U^{\rho}} \widetilde{W}^{\sigma}-\mathrm{D}_{i}\left(\frac{\partial \Lambda_{\sigma}[U]}{\partial U_{i}^{\rho}} \widetilde{W}^{\sigma}\right) \\
& \quad+\ldots+(-1)^{k} \mathrm{D}_{i_{1}} \ldots \mathrm{D}_{i_{l}}\left(\frac{\partial \Lambda_{\sigma}[U]}{\partial U_{i_{1} \ldots i_{l}}^{\rho}} \widetilde{W}^{\sigma}\right), \quad \rho=1, \ldots, m
\end{aligned}
$$

acting respectively on arbitrary functions $\widetilde{V}=\left(\widetilde{V}^{1}(x), \ldots, \widetilde{V}^{m}(x)\right)$ and $\widetilde{W}=\left(\widetilde{W}_{1}(x), \ldots, \widetilde{W}_{N}(x)\right)$.

It is straightforward to show that the operators (A.1) - A.4 satisfy the following divergence identities:

$$
\begin{aligned}
& W_{\sigma}\left(\mathrm{L}_{R}\right)_{\rho}^{\sigma}[U] V^{\rho}-V^{\rho}\left(\mathrm{L}_{R}^{*}\right)_{\rho}^{\sigma}[U] W_{\sigma} \equiv \mathrm{D}_{i} S^{i}[V, W ; R[U]] \\
& \widetilde{W}^{\sigma}\left(\mathrm{L}_{\Lambda}\right)_{\sigma \rho}[U] \widetilde{V}^{\rho}-\widetilde{V}^{\rho}\left(\mathrm{L}_{\Lambda}^{*}\right)_{\sigma \rho}[U] \widetilde{W}^{\sigma} \equiv \mathrm{D}_{i} \widetilde{S^{i}}[\widetilde{V}, \widetilde{W} ; \Lambda[U]]
\end{aligned}
$$

where operators $S^{i}, \widetilde{S}^{i}$ are defined by (4.21) and (4.22).

To avoid the appearance of singular integrals, we need the following construction. Let

$$
U_{(\lambda)} \equiv U+(\lambda-1) V
$$


where $U=\left(U^{1}(x), \ldots, U^{m}(x)\right)$ and $\left.V=V^{1}(x), \ldots, V^{m}(x)\right)$ are arbitrary functions, and $\lambda$ is a scalar parameter. Replacing $U$ by $U_{(\lambda)}$ in the conservation law identity (3.1), one has

$$
\frac{\partial}{\partial \lambda}\left(\Lambda_{\sigma}\left[U_{(\lambda)}\right] R^{\sigma}\left[U_{(\lambda)}\right]\right) \equiv \frac{\partial}{\partial \lambda} \mathrm{D}_{i} \Phi^{i}\left[U_{(\lambda)}\right]=\mathrm{D}_{i}\left(\frac{\partial}{\partial \lambda} \Phi^{i}\left[U_{(\lambda)}\right]\right)
$$

(The last identity holds since $\lambda$ can be viewed as an additional independent variable.) The left-hand side of (A.8) can then be expressed in terms of the linearizing operators (A.1) and (A.3) as follows:

$$
\frac{\partial}{\partial \lambda}\left(\Lambda_{\sigma}\left[U_{(\lambda)}\right] R^{\sigma}\left[U_{(\lambda)}\right]\right)=\Lambda_{\sigma}\left[U_{(\lambda)}\right]\left(\mathrm{L}_{R}\right)_{\rho}^{\sigma}\left[U_{(\lambda)}\right] V^{\rho}+R^{\sigma}\left[U_{(\lambda)}\right]\left(\mathrm{L}_{\Lambda}\right)_{\sigma \rho}\left[U_{(\lambda)}\right] V^{\rho} .
$$

From (A.5) and (A.6) with $W_{\sigma}=\Lambda_{\sigma}\left[U_{(\lambda)}\right]$ and $\widetilde{W}^{\sigma}=R^{\sigma}\left[U_{(\lambda)}\right]$, respectively, and using the property

$$
\left(\mathrm{L}_{R}^{*}\right)_{\rho}^{\sigma}[U] \Lambda_{\sigma}[U]+\left(\mathrm{L}_{\Lambda}^{*}\right)_{\sigma \rho} R^{\sigma}[U]=0
$$

of the adjoint operators (A.2), (A.4), one can show that

$$
\begin{aligned}
\frac{\partial}{\partial \lambda}\left(\Lambda_{\sigma}\left[U_{(\lambda)}\right] R^{\sigma}\left[U_{(\lambda)}\right]\right)= & V^{\rho}\left(\mathrm{L}_{R}^{*}\right)_{\rho}^{\sigma}\left[U_{(\lambda)}\right] \Lambda_{\sigma}\left[U_{(\lambda)}\right]+\mathrm{D}_{i} S^{i}\left[V, \Lambda\left[U_{(\lambda)}\right] ; R\left[U_{(\lambda)}\right]\right] \\
& +V^{\rho}\left(\mathrm{L}_{\Lambda}^{*}\right)_{\sigma \rho}\left[U_{(\lambda)}\right] R^{\sigma}\left[U_{(\lambda)}\right]+\mathrm{D}_{i} \widetilde{S}^{i}\left[V, R\left[U_{(\lambda)}\right] ; \Lambda\left[U_{(\lambda)}\right]\right] \\
= & \mathrm{D}_{i}\left(S^{i}\left[V, \Lambda\left[U_{(\lambda)}\right] ; R\left[U_{(\lambda)}\right]\right]+\widetilde{S}^{i}\left[V, R\left[U_{(\lambda)}\right] \Lambda\left[U_{(\lambda)}\right]\right]\right) .
\end{aligned}
$$

Comparing (A.8) and (A.9), one finds that

$$
\mathrm{D}_{i}\left(\frac{\partial}{\partial \lambda} \Phi^{i}\left[U_{(\lambda)}\right]\right)=\mathrm{D}_{i}\left(S^{i}\left[V, \Lambda\left[U_{(\lambda)}\right] ; R\left[U_{(\lambda)}\right]\right]+\widetilde{S}^{i}\left[V, R\left[U_{(\lambda)}\right] ; \Lambda\left[U_{(\lambda)}\right]\right]\right)
$$

which implies

$$
\frac{\partial}{\partial \lambda} \Phi^{i}\left[U_{(\lambda)}\right]=S^{i}\left[V, \Lambda\left[U_{(\lambda)}\right] ; R\left[U_{(\lambda)}\right]\right]+\widetilde{S}^{i}\left[V, R\left[U_{(\lambda)}\right] ; \Lambda\left[U_{(\lambda)}\right]\right]
$$

up to fluxes of a trivial conservation law. Now let $V=U-\widetilde{U}$, for an arbitrary function $\widetilde{U}=$

$\left(\widetilde{U}^{1}(x), \ldots, \widetilde{U}^{m}(x)\right)$. Then $U_{(\lambda)}=\lambda U+(1-\lambda) \widetilde{U}$. Integrating (A.10) with respect to $\lambda$ from 0 to 1 , one obtains the desired expression (4.20).

\section{B Computation of fluxes of a conservation law from a symme- try/adjoint symmetry pair}

Suppose a given PDE system (2.1) has a local symmetry with generator (in the characteristic form)

$$
\hat{\mathrm{X}}=\hat{\eta}^{\rho}[u] \frac{\partial}{\partial u^{\rho}} .
$$

The symmetry components $\hat{\eta}^{\rho}[u]$ are solutions of the symmetry determining equations, i.e., the linearized system

$$
\mathrm{L}_{\rho}^{\sigma}[u] \hat{\eta}^{\rho}[u]=0,
$$

with $\mathrm{L}_{\rho}^{\sigma} \equiv\left(\mathrm{L}_{R}\right)_{\rho}^{\sigma}$ given by (A.1). 
Similarly, let $\left\{\omega_{\sigma}[u]\right\}_{\sigma=1}^{N}$ be some solution of the adjoint linearized system

$$
\mathrm{L}_{\rho}^{* \sigma}[u] \omega_{\sigma}[u]=0
$$

with $\mathrm{L}_{\rho}^{* \sigma} \equiv\left(\mathrm{L}_{R}^{*}\right)_{\rho}^{\sigma}$ given by (A.2). (Functions $\left\{\omega_{\sigma}[u]\right\}_{\sigma=1}^{N}$ are often called adjoint symmetries).

The linearizing operator (A.1) and its adjoint (A.2) are related through a well-known formula [16]

$$
W_{\sigma} \mathrm{L}_{\rho}^{\sigma}[U] V^{\rho}-V^{\rho} \mathrm{L}_{\rho}^{* \sigma}[U] W_{\sigma} \equiv \mathrm{D}_{i} \Psi^{i}[U]
$$

where $\Psi^{i}[U]$ are given by

$$
\Psi^{i}[U]=\sum_{p=0}^{k-1} \sum_{q=0}^{k-p-1}(-1)^{q}\left(\mathrm{D}_{i_{1}} \ldots \mathrm{D}_{i_{p}} V^{\rho}\right) \mathrm{D}_{j_{1}} \ldots \mathrm{D}_{j_{q}}\left(W_{\sigma} \frac{\partial R^{\sigma}[U]}{\partial U_{j_{1} \ldots j_{q} i i_{1} \ldots i_{p}}^{\rho}}\right)
$$

where $j_{1} \ldots j_{q}$ and $i_{1} \ldots i_{p}$ are ordered combinations of indices such that $1 \leq j_{1} \leq \ldots \leq j_{q} \leq i \leq$ $i_{1} \leq \ldots \leq i_{p} \leq n$.

The following result is immediately obtained.

Theorem 7. For a given PDE system (2.1), let $\left\{\hat{\eta}^{\rho}[u]\right\}_{\rho=1}^{m}$ be components of its local symmetry (B.1), and $\left\{\omega_{\sigma}[u]\right\}_{\sigma=1}^{N}$ a solution of the adjoint linearized system (B.3). Then formulas (4.29) with $V^{\rho}=\eta^{\rho}[U]$ and $W_{\sigma}=\omega_{\sigma}[U]$ yield fluxes of local conservation laws of the PDE system (2.1).

The proof follows from noting that for the symmetry/adjoint symmetry pair $\left\{\hat{\eta}^{\rho}[u]\right\}_{\rho=1}^{m},\left\{\omega_{\sigma}[u]\right\}_{\sigma=1}^{N}$, using $V^{\rho}=\hat{\eta}^{\rho}[u]$ and $W_{\sigma}=\omega_{\sigma}[u]$ in formulas (B.4) yields $\mathrm{D}_{i} \Psi^{i}[u]=0$, which is a local conservation laws of the PDE system (2.1).

The following remarks are important.

1. Since every set of local conservation law multipliers $\left\{\Lambda_{\sigma}[U]\right\}_{\sigma=1}^{N}$ of a given PDE system (2.1) satisfies the adjoint linearized equations (B.3), it follows that in Theorem 7 , one can use any local symmetry and any set of local conservation law multipliers of a given PDE system to generate its local conservation law. (Note in some cases, it is possible that a so generated conservation law is trivial.)

2. A conservation law with fluxes $\Psi^{i}[U]$ (4.29), obtained from a symmetry/multiplier pair using Theorem 17, is generally inequivalent to the conservation law (3.2) with fluxes $\Phi^{i}[U]$, which corresponds to the multipliers $\left\{\Lambda_{\sigma}[U]\right\}_{\sigma=1}^{N}$. One obtains the fluxes corresponding to the multipliers only in the case of a scaling symmetry, as discussed in Section 4.4 .

\section{References}

[1] Lax P.D., "Integrals of nonlinear equations of evolution and solitary waves." Comm. Pure and Appl. Math. 21, 467-490 (1968).

[2] Benjamin T.B., "The stability of solitary waves." Proc. Roy. Soc. Lond. A 328, 153-183 (1972).

[3] Knops R.J. and Stuart C.A., "Quasiconvexity and uniqueness of equilibrium solutions in nonlinear elasticity." Arch. Rat. Mech. Anal. 86, 234-249 (1984).

[4] LeVeque R.J.Numerical Methods for Conservation Laws. Birkhäuser (1992). 
[5] Godlewski E. and Raviart P.-A. Numerical Approximation of Hyperbolic Systems of Conservation Laws. Springer (1996).

[6] Bluman, G and Kumei, S, On invariance properties of the wave equation, J. Math. Phys. 28, 307-318 (1987).

[7] Bluman, G, Kumei, S and Reid, G, New classes of symmetries for partial differential equations, J. Math. Phys. 29, 806-811 (1988).

[8] Bluman G. and Cheviakov A. F., Framework for potential systems and nonlocal symmetries: Algorithmic approach. J. Math. Phys. 46, 123506 (2005).

[9] Bluman G., Cheviakov A. F., and Ivanova N.M., Framework for nonlocally related PDE systems and nonlocal symmetries: extension, simplification, and examples. J. Math. Phys. 47, 113505 (2006).

[10] Sjöberg A. and Mahomed F.M., Non-local symmetries and conservation laws for one-dimensional gas dynamics equations. App. Math. Comp. 150, 379397 (2004).

[11] Akhatov S., Gazizov R and Ibragimov N., Nonlocal symmetries. Heuristic approach. J. Sov. Math. 55, 1401-1450 (1991).

[12] Anco S.C., Bluman, G.W., and Wolf T. Invertible mappings of nonlinear PDEs to linear PDEs through admitted conservation laws. Acta Appl. Math. 101, 21-38 (2008).

[13] Noether, E. Invariante Variationsprobleme. Nachr. König. Gesell. Wissen. Göttingen, Math.-Phys. Kl., pp. 235-257 (1918).

[14] Bluman G. Connections Between Symmetries and Conservation Laws. Symm. Integr. E Geom.: Meth. Appl. (SIGMA) 1 , Paper 011, 16 pages (2005).

[15] Wolf T., "A comparison of four approaches to the calculation of conservation laws." Europ. J. Appl. Math. 13 (2) 129-152, (2002).

[16] Anco S. and Bluman G., Direct construction of conservation laws. Phys. Rev. Lett. 78, 2869-2873 (1997).

[17] Anco S. and Bluman G., Direct construction method for conservation laws of partial differential equations Part II: General treatment. Europ. J. Appl. Math. 13, 567-585 (2002).

[18] Bluman G., Cheviakov A.F., and Anco S. Construction of Conservation Laws: How the Direct Method Generalizes Noether's Theorem. To appear in Proceedings of 4 th Workshop "Group Analysis of Differential Equations \& Integrability" (2009).

[19] Hereman W., Colagrosso M., Sayers R., Ringler A., Deconinck B., Nivala M., and Hickman M.S. Continuous and discrete homotopy operators and the computation of conservation laws. In: Differential Equations with Symbolic Computation, D. Wang and Z. Zheng, eds., Birkhäuser Verlag, 249-285 (2005).

[20] Anco S. Conservation laws of scaling-invariant field equations. J. Phys. A: Math. Gen. 36, 8623-8638 (2003).

[21] Bluman G.W, Cheviakov A.F. and Anco S.C., Advanced Symmetry Methods for Partial Differential Equations. To appear in the Applied Mathematical Sciences series, Springer (2009).

[22] Cheviakov A. F., Comp. Phys. Comm. 176 (1), 48-61 (2007). (In the current paper, we used a new version of GeM software, which is scheduled for public release in 2009. See http://math.usask.ca/ cheviakov/gem/)

[23] Olver P.J. Conservation laws and null divergences. Math. Proc. Camb. Phil. Soc. 94, 529-540 (1983).

[24] Oberlack M., Wenzel H., and Peters N. On symmetries and averaging of the G-equation for premixed combustion. Combust. Theory Modelling 5, 363-383 (2001).

[25] Oberlack M. and Cheviakov A.F. Higher-order symmetries and conservation laws of the G-equation for premixed combustion and resulting numerical schemes. Submitted to J. Eng. Math. (2009).

[26] Wolf T., "Crack, LiePDE, ApplySym and ConLaw, section 4.3.5 and computer program on CD-ROM" in: Grabmeier, J., Kaltofen, E. and Weispfenning, V. (Eds.): Computer Algebra Handbook, Springer, pp. 465-468 (2002).

[27] Hereman W., TransPDEDensityFlux.m, PDEMultiDimDensityFlux.m, and DDEDensityFlux.m: Mathematica packages for the symbolic computation of conservation laws of partial differential equations and differentialdifference equations. Available from the software section at http://www.mines.edu/fs_home/whereman/. 
[28] Deconinck B. and Nivala M., Symbolic integration using homotopy methods, Preprint, Department of Applied Mathematics, University of Washington, Seattle, WA 98195-2420; Math. Comput. Simulat. (in press) (2008).

[29] Deconinck B. and Nivala M., Maple software for the symbolic computation of conservation laws of $(1+1)-$ dimensional partial differential equations. Available at

http://www. amath. washington. edu/ ־ bernard/papers.html. 